\title{
An Efficient Approach for SIMO Systems using Adaptive Fuzzy Hierarchical Sliding Mode Control
}

\author{
Thien Van Nguyen and Hai Xuan Le \\ Hanoi University of Industry \\ Hanoi, Vietnam \\ nguyenthien@haui.edu.vn; hailx@haui.edu.vn \\ Minh Ngoc Nguyen \\ Hanoi University of Science and Technology \\ Hanoi, Vietnam \\ minhkenzin123@gmail.com
}

\author{
Hoang Viet Tran and Duc Anh Nguyen \\ Hanoi University of Science and Technology \\ Hanoi, Vietnam \\ \{hoangprohp1999,anhduc180198\}@gmail.com \\ Linh Nguyen \\ Federation University Australia \\ VIC, Australia \\ 1.nguyen@federation.edu.au
}

\begin{abstract}
The paper addresses the problem of efficiently controlling a class of single input multiple output (SIMO) underactuated robotic systems such as a two dimensional inverted pendulum cart or a two dimensional overhead crane. It is first proposed to employ the hierarchical sliding mode control approach to design a control law, which guarantees stability and anti-swing of the vehicle when it is driven on a predefined trajectory. More importantly, the unknown and uncertain parameters of the system caused by its actuator nonlinearity and external disturbances are adaptively estimated and inferred by the proposed fuzzy logic mechanism, which results in the efficient operation of the SIMO under-actuated system in real time. The proposed algorithm was then implemented in the synthetic environment, where the obtained results demonstrate its effectiveness.
\end{abstract}

Index Terms-Adaptive control, sliding mode control, fuzzy logic, under-actuated systems, Lyapunov theory

\section{INTRODUCTION}

Under-actuated robotic systems have been playing a crucial role in many applications from industries or factories to transport and space and underwater exploration [1]. Depending on the number of input signals, an under-actuated robotic system can be considered as single input multiple output (SIMO) or multiple input multiple output mechanisms [2]. A SIMO under-actuated robotic system such as a two dimensional inverted pendulum cart or a two dimensional overhead crane will be discussed in this work. Having more outputs than inputs, a SIMO system is constrained by strong nonlinearities, internal and external uncertainties and coupling properties. Hence, efficiently controlling an under-actuated robotic system with fewer independent control signals than its degrees of freedom is a fundamental but still challenging problem.

Due to its fundamentality, researchers, engineers and practitioners who design a control law for a SIMO under-actuated robotic system always concern about robustness in the system response due to its parameter uncertainties and actuator nonlinearities. To address the concern, sliding mode control (SMC) method has been then favoured for those systems [3]-[6]. For instance, the authors in [7]-[9] proposed the robust SMC controllers for a gantry crane, which allow the system with uncertain parameters and nonlinear actuators to robustly work under external disturbances in a working environment. It is noted that challenge in designing a robust SMC scheme for a SIMO under-actuated system is how to formulate sliding surfaces. In the work [10], the authors proposed to compute an intermediate variable from the state errors before a second-level sliding surface is formulated. Recently, the hierarchical SMC (HSMC) approach has been frequently utilized in defining the sliding surface for the SMC scheme in applications using under-actuated robotic systems [8], [9], [11]. For instance, Wang et al. in their work [11] defined a first-level sliding surface for each subsystem of a a second-order under-actuated system. They then established the second-level sliding surface by simply adding the first-level sliding surfaces together.

Nonetheless, when developing a HSMC strategy for a SIMO under-actuated system, we have learned that if some parameters in the HSMC scheme are not properly chosen, it can severely causes vibration in the control system. Choosing an improper parameter for the HSMC controller is probably due to imprecision and imperfect information in the SIMO under-actuated system. That is very likely in practice since the SIMO under-actuated robotic systems are constrained by their highly complicated nonlinearities and uncertainties. So far, it is well-known that fuzzy logic has been frequently exploited in addressing issues of inferring parameters for highly uncertain control systems [12]. For instance, the fuzzy logic has been extensively employed in controlling under-actuated systems [13].

In this work, we take advantages of the HSMC approach, which guarantees robustness in the control performance, to design a controller for a SIMO under-actuated robotic system. It is assumed that the parameters in the HSMC law are deterministic. Nonetheless, in fact due to the nonlinear and uncertain properties, determining those parameters in the SIMO under-actuated robotic system is impractical. Moreover, if those parameters are not properly chosen, it can lead 
to severe vibration in the control system. To this end, we propose to exploit the fuzzy logic to adaptively infer the parameters of the HSMC scheme over time. It is noted that the fuzzy logic can effectively estimate the parameters of a system given its imprecision and imperfect information. More importantly, stability of the control performance in the SIMO under-actuated robotic system obtained by the proposed approach is theoretically proved by the Lyapunov theory. We then evaluated our algorithm in two typical SIMO underactuated robotic systems including the 2D inverted pendulum cart and 2D overhead crane, where the obtained results are highly promising.

The remaining of the paper is organized as follows. Section II presents two typical models in the class of a SIMO underactuated robotic system, which comprise a 2D inverted pendulum cart and a 2D overhead crane. We then introduce how to design an adaptive controller by exploiting the HSMC and fuzzy logic in Section III. The demonstrated results obtained by the proposed approach are presented and discussed in Section IV before the conclusions are drawn in Section V.

\section{Single Input Multiple Output Models}

In this section, we present two typical models in the class of SIMO under-actuated nonlinear systems including a 2D inverted pendulum cart and a $2 \mathrm{D}$ overhead crane. These models will be then utilized to evaluate effectiveness of our proposed approach.

\section{A. 2D Inverted Pendulum Cart System}

Let us consider a 2D inverted pendulum model [14]. For the simplicity purpose, it is assumed that there is no friction in the system. In this work, we denote notations in the inverted pendulum system as follows. $l$ is the length of the pendulum and $I$ is the moment of inertia to center of the pendulum while $\theta$ is the angle between the pendulum and the vertical. $l_{c}$ is defined as the distance from the origin to center of the pendulum; and the control force is denoted by $u . m_{0}$ and $m$ are the masses of the cart and the pendulum, respectively.

Mathematically, a model of a 2D inverted pendulum can be presented by

$$
\begin{aligned}
& x_{g}=x+l_{c} \sin \theta \quad y_{g}=l_{c} \cos \theta \\
& \dot{x}_{g}=\dot{x}+l_{c} \dot{\theta} \cos \theta \quad \dot{y}_{g}=-l_{c} \dot{\theta} \sin \theta \\
& \ddot{x}_{g}=\ddot{x}-l_{c} \dot{\theta}^{2} \sin \theta+l_{c} \ddot{\theta} \cos \theta \\
& \ddot{y}_{g}=-l_{c} \dot{\theta}^{2} \cos \theta-l_{c} \ddot{\theta} \sin \theta,
\end{aligned}
$$

where $\left(x_{g}, y_{g}\right),\left(\dot{x}_{g}, \dot{y}_{g}\right)$ and $\left(\ddot{x}_{g}, \ddot{y}_{g}\right)$ are the position, velocity and acceleration of the pendulum, respectively, while $x, \dot{x}$ and $\ddot{x}$ are the counterparts applied to the cart.

Under influence of the control force $u$, the motions of both the cart and pendulum are specified as follows,

$$
u-N_{x}=m_{0} \ddot{x} \quad N_{x}=m \ddot{x}_{g} .
$$

From (1), it yields

$$
u-N_{x}=u-m\left(\ddot{x}-l_{c} \dot{\theta}^{2} \sin \theta+l_{c} \ddot{\theta} \cos \theta\right)=m_{0} \ddot{x} .
$$

Then

$$
\ddot{x}=\frac{u+m l_{c} \dot{\theta}^{2} \sin \theta-m l_{c} \ddot{\theta} \cos \theta}{m_{0}+m} .
$$

On the other hand, the equilibrium torque of the pendulum is given by

$$
I_{0} \ddot{\theta}=-F_{q t} l_{c} \cos \theta+m g l_{c} \sin \theta,
$$

where

$$
\begin{aligned}
& I_{0}=I+m l_{c}{ }^{2}=\frac{4}{3} m l_{c}{ }^{2} \\
& F_{q t}=m . \ddot{x}=m \frac{u+m l_{c} \dot{\theta}^{2} \sin \theta-m l_{c} \ddot{\theta} \cos \theta}{m_{0}+m},
\end{aligned}
$$

and $g$ is the gravitational acceleration, which leads to

$$
\begin{aligned}
\left(I+m l_{c}{ }^{2}\right) \ddot{\theta}= & -m \frac{u+m l_{c} \dot{\theta}^{2} \sin \theta-m l_{c} \ddot{\theta} \cos \theta}{m_{0}+m} l_{c} \cos \theta \\
& +m g l_{c} \sin \theta .
\end{aligned}
$$

Therefore,

$$
\begin{aligned}
& \ddot{x}=\frac{\left(u+m l_{c} \dot{\theta}^{2} \sin \theta\right)\left(I+m l_{c}^{2}\right)-m^{2} l_{c}^{2} g \sin \theta \cos \theta}{\left(m_{0}+m\right)\left(I+m l_{c}^{2}\right)-m^{2} l_{c}{ }^{2} \cos ^{2} \theta} \\
& \ddot{\theta}=\frac{\left(m_{0}+m\right) m l_{c} g \sin \theta-m l_{c} \cos \theta\left(u+m l_{c} \dot{\theta}^{2} \sin \theta\right)}{\left(m_{0}+m\right)\left(I+m l_{c}{ }^{2}\right)-m^{2} l_{c}{ }^{2} \cos ^{2} \theta} .
\end{aligned}
$$

If we define $x_{1}=x, x_{2}=\dot{x}, x_{3}=\theta$ and $x_{4}=\dot{\theta}$, then

$$
\begin{aligned}
& \dot{x}_{1}=x_{2} \\
& \dot{x}_{2}=\frac{\left(u+m l_{c} \sin x_{3} x_{4}{ }^{2}\right)\left(I+m l_{c}^{2}\right)-m^{2} l_{c}^{2} g \sin x_{3} \cos x_{3}}{\left(m_{0}+m\right)\left(I+m l_{c}{ }^{2}\right)-m^{2} l_{c}{ }^{2} \cos ^{2} x_{3}} \\
& \dot{x}_{3}=x_{4} \\
& \dot{x}_{4}=\frac{-m l_{c}\left(\cos x_{3}\left(u+m l_{c} \sin x_{3} x_{4}{ }^{2}\right)+\left(m_{0}+m\right) g \sin x_{3}\right)}{\left(m_{0}+m\right)\left(I+m l_{c}{ }^{2}\right)-m^{2} l_{c}{ }^{2} \cos ^{2} x_{3}}
\end{aligned}
$$

For simplicity in the presentation in designing a controller for the inverted pendulum, (7) can be rewritten as follows,

$$
\begin{array}{ll}
\dot{x}_{1}=x_{2} & \dot{x}_{2}=f_{1}(X)+g_{1}(X) u \\
\dot{x}_{3}=x_{4} & \dot{x}_{4}=f_{2}(X)+g_{2}(X) u,
\end{array}
$$

where

$$
\begin{aligned}
f_{1}(X) & =\frac{\left(m l_{c} \sin x_{3} x_{4}^{2}\right)\left(I+m l_{c}^{2}\right)-m^{2} l_{c}^{2} g \sin x_{3} \cos x_{3}}{\left(m_{0}+m\right)\left(I+m l_{c}^{2}\right)-m^{2} l_{c}^{2} \cos ^{2} x_{3}} \\
g_{1}(X) & =\frac{\left(I+m l_{c}^{2}\right)}{\left(m_{0}+m\right)\left(I+m l_{c}^{2}\right)-m^{2} l_{c}^{2} \cos ^{2} x_{3}} \\
f_{2}(X) & =\frac{m l_{c}\left(\left(m_{0}+m\right) g \cdot \sin x_{3}-\cos x_{3}\left(m l_{c} \sin x_{3} x_{4}^{2}\right)\right)}{\left(m_{0}+m\right)\left(I+m l_{c}^{2}\right)-m^{2} l_{c}^{2} \cos ^{2} x_{3}} \\
g_{2}(X) & =\frac{-m l_{c} \cos x_{3}}{\left(m_{0}+m\right)\left(I+m l_{c}^{2}\right)-m^{2} l_{c}{ }^{2} \cos ^{2} x_{3}} .
\end{aligned}
$$

\section{B. 2D Overhead Crane}

Another typical SIMO under-actuated system is a 2D overhead crane [9]. In this model, we denote $l$ as the length of the hoisting cable. While $u$ is the control force, $M_{0}$ and $m_{c}$ are the masses of the trolley and payload, respectively. The angle between the hoisting cable and the vertical is defined by $\varphi$. 
Similar to the 2D inverted pendulum mechanism, the $2 \mathrm{D}$ overhead crane is mathematically presented by

$$
\left\{\begin{array}{l}
\left(M_{0}+m_{c}\right) \ddot{x}+m_{c} l \ddot{\varphi} \cos (\varphi)-m_{c} l \dot{\varphi}^{2} \sin (\varphi)=u \\
l \ddot{\varphi}+g \sin (\varphi)+\ddot{x} \cos (\varphi)=0,
\end{array}\right.
$$

where $\ddot{x}$ is the acceleration of the trolley and $g$ is also the gravitational acceleration. Hence,

$$
\begin{gathered}
\ddot{x}=\frac{u-m_{c} l \ddot{\varphi} \cos (\varphi)+m_{c} l \dot{\varphi}^{2} \sin (\varphi)}{M_{0}+m_{c}} \\
\ddot{\varphi}=\frac{-\ddot{x} \cos (\varphi)-g \sin (\varphi)}{l} .
\end{gathered}
$$

From (10) and (12), one has

$$
\begin{aligned}
u= & \left(M_{0}+m_{c}\right) \ddot{x}+m_{c} l \cos (\varphi) \frac{-\ddot{x} \cos (\varphi)-g \sin (\varphi)}{l} \\
& -m_{c} l \dot{\varphi}^{2} \sin (\varphi) .
\end{aligned}
$$

In other words,

$$
\ddot{x}=\frac{m_{c} g \cos (\varphi) \sin (\varphi)+m_{c} l \dot{\varphi}^{2} \sin (\varphi)}{M_{0}+m_{c} \sin ^{2}(\varphi)}+\frac{u}{M_{0}+m_{c} \sin ^{2}(\varphi)} \text {. }
$$

Likewise, if substituting (11) in (10), it yields

$$
\frac{u-m_{c} l\left(\ddot{\varphi} \cos (\varphi)+\dot{\varphi}^{2} \sin (\varphi)\right)}{M_{0}+m_{c}} \cos (\varphi)+l \ddot{\varphi}+g \sin (\varphi)=0,
$$

and then

$$
\begin{aligned}
& \left(M_{0}+m_{c}\right) l \ddot{\varphi}+\left(M_{0}+m_{c}\right) g \sin (\varphi)+\left(u-m_{c} l \ddot{\varphi} \cos (\varphi)\right. \\
& \left.+m_{c} l \dot{\varphi}^{2} \sin (\varphi)\right) \cos (\varphi)=0,
\end{aligned}
$$

which results in

$$
\ddot{\varphi}=-\frac{\left(\left(M_{0}+m_{c}\right) g+m_{c} l \dot{\varphi}^{2} \cos (\varphi)\right) \sin (\varphi)-\cos (\varphi) u}{\left(M_{0}+m_{c} \sin ^{2}(\varphi)\right) l}
$$

Thus, the model of the 2D overhead crane (10) can be rewritten by

$$
\left\{\begin{array}{l}
\ddot{x}=\frac{\left(m_{c} g \cos (\varphi)+m_{c} l \dot{\varphi}^{2}\right) \sin (\varphi)}{M_{0}+m_{c} \sin ^{2}(\varphi)}+\frac{u}{M_{0}+m_{c} \sin ^{2}(\varphi)} \\
\ddot{\varphi}=-\frac{\left(\left(M_{0}+m_{c}\right) g+m_{c} l \dot{\varphi}^{2} \cos (\varphi)\right) \sin (\varphi)-\cos (\varphi) u}{\left(M_{0}+m_{c} \sin ^{2}(\varphi)\right) l} .
\end{array}\right.
$$

If we define $x_{1}=x, x_{2}=\dot{x}, x_{3}=\varphi$ and $x_{4}=\dot{\varphi}$, (16) can be represented in a simple form as follows,

$$
\begin{array}{ll}
\dot{x}_{1}=x_{2} & \dot{x}_{2}=f_{1}(X)+g_{1}(X) u \\
\dot{x}_{3}=x_{4} & \dot{x}_{4}=f_{2}(X)+g_{2}(X) u,
\end{array}
$$

where

$$
\begin{aligned}
& f_{1}(X)=\frac{m_{c} g \cos \left(x_{3}\right) \sin \left(x_{3}\right)+m_{c} l x_{4}^{2} \sin \left(x_{3}\right)}{M_{0}+m_{c} \sin ^{2}\left(x_{3}\right)} \\
& g_{1}(X)=\frac{1}{M_{0}+m_{c} \sin ^{2}\left(x_{3}\right)} \\
& f_{2}(X)=-\frac{\left(M_{0}+m_{c}\right) g \sin \left(x_{3}\right)+m_{c} l x_{4}^{2} \sin \left(x_{3}\right) \cos \left(x_{3}\right)}{\left(M_{0}+m_{c} \sin ^{2}\left(x_{3}\right)\right) l} \\
& g_{2}(X)=\frac{-\cos \left(x_{3}\right)}{\left(M_{0}+m_{c} \sin ^{2}\left(x_{3}\right)\right) l} .
\end{aligned}
$$

Given the two described models representing for a class of SIMO systems, we now design a controller to robustly and effectively control those systems.

\section{Adaptive Fuzzy Hierarchical Sliding Mode CONTROL SCHEME}

In this section, we will present how to design a HSMC scheme for a SIMO under-actuated system and then introduce an adaptive law in the controller, which enables the SIMO under-actuated system to robustly and effectively deal with its inherent nonlinearities and uncertainties.

\section{A. Controller Design}

Let us consider the typical model of a class of a SIMO under-actuated system as presented in Section II as follows,

$$
\begin{array}{ll}
\dot{x}_{1}=x_{2} & \dot{x}_{2}=f_{1}(X)+g_{1}(X) u \\
\dot{x}_{3}=x_{4} & \dot{x}_{4}=f_{2}(X)+g_{2}(X) u .
\end{array}
$$

The errors between the system responses and the desired references are

$$
e(t)=\left[\begin{array}{l}
x_{1}-x_{d} \\
x_{3}-\varphi_{d}
\end{array}\right]=\left[\begin{array}{l}
x_{1}-x_{d} \\
x_{3}-0
\end{array}\right]=\left[\begin{array}{l}
e_{1} \\
e_{3}
\end{array}\right],
$$

where $x_{d}$ is the desired position. In the cases of the inverted pendulum and overhead crane, $x_{d}$ is the desired position of the cart or trolley. $\varphi_{d}=0$ is the desired swing angle. It is supposed that there exist $\dot{x}_{d}$ and $\ddot{x}_{d}$, which are bounded; therefore, derivatives of the errors can be derived from (18) as follows,

$$
\begin{array}{ll}
\dot{e}_{1}=e_{2} & \dot{e}_{2}=f_{1}(X)+g_{1}(X) u-\ddot{x}_{d} \\
\dot{e}_{3}=e_{4} & \dot{e}_{4}=f_{2}(X)+g_{2}(X) u .
\end{array}
$$

Two first-level sliding surfaces are first defined as follows,

$$
s_{1}=c_{1} e_{1}+e_{2} \quad s_{2}=c_{2} e_{3}+e_{4},
$$

where $c_{1}$ and $c_{2}$ are positive constants. Then the secondlevel sliding surface for the SIMO under-actuated systems is summarized by

$$
S=\lambda_{1} s_{1}+\beta_{1} s_{2}
$$

where $\lambda_{1}$ and $\beta_{1}$ are also positive parameters. Hence, the efficient control scheme for a SIMO under-actuated system is formulated as follows,

$$
u=u_{e q}+u_{s w},
$$

where $u_{e q}$ and $u_{s w}$ are the equivalent control and switch control laws, respectively. Moreover, in order to ensure stability of the SIMO under-actuated system under the proposed control scheme, a Lyapunov function candidate is considered by

$$
V=\frac{1}{2} S^{2}
$$


For further analysis, let us compute derivative of the secondlevel sliding surface as follows,

$$
\begin{aligned}
& \frac{d V}{d t}=S \dot{S} \\
& =S\left[\lambda_{1} \dot{s}_{1}+\beta_{1} \dot{s}_{2}\right] \\
& =S\left[\lambda_{1}\left(c_{1} x_{2}+f_{1}+g_{1} u\right)+\beta_{1}\left(c_{2} x_{4}+f_{2}+g_{2} u\right)\right] \\
& =S\left[\begin{array}{l}
\lambda_{1}\left(c_{1} x_{2}+f_{1}+g_{1}\left(u_{e q 1}+u_{s \mathrm{w} 1}+u_{e q 2}+u_{s \mathrm{w} 2}\right)\right) \\
+\beta_{1}\left(c_{2} x_{4}+f_{2}+g_{2}\left(u_{e q 1}+u_{s \mathrm{w} 1}+u_{e q 2}+u_{s \mathrm{w} 2}\right)\right)
\end{array}\right. \\
& =S\left[\begin{array}{l}
\lambda_{1}\left(c_{1} x_{2}+f_{1}+g_{1} u_{e q 1}\right)+\beta_{1}\left(c_{2} x_{4}+f_{2}+g_{2} u_{e q 2}\right) \\
+u_{s w 2}\left(\lambda_{1} g_{1}+\beta_{1} g_{2}\right)+u_{s w 1}\left(\lambda_{1} g_{1}+\beta_{1} g_{2}\right) \\
+\lambda_{1} g_{1} u_{e q 2}+\beta_{1} g_{2} u_{e q 1}+k S+\eta \operatorname{sat}(S) \\
-(k S+\eta \operatorname{sgn}(S)),
\end{array}\right]
\end{aligned}
$$

where $\operatorname{sat}(\cdot)$ and $\operatorname{sgn}(\cdot)$ are the saturation and sign functions, respectively. $k$ is the parameter that can be used to eliminate the chattering phenomena; and, $u_{e q}=u_{e q 1}+u_{e q 2}, u_{s \mathrm{w}}=$ $u_{s \mathrm{w} 1}+u_{s \mathrm{w} 2}$. It is noted that stability of the sliding surface $S$ is guaranteed if

$$
\left\{\begin{array}{l}
u_{e q 1}=\frac{-\left(c_{1} x_{2}+f_{1}\right)}{g_{1}} \\
u_{e q 2}=\frac{-\left(c_{2} x_{4}+f_{2}\right)}{g_{2}} \\
u_{s w 2}=-u_{s w 1}-\frac{\lambda_{1} g_{1} u_{e q 1}+\beta_{1} g_{2} u_{e q 2}}{\lambda_{1} g_{1}+\beta_{1} g_{2}}-\frac{k \cdot S+\eta \cdot \operatorname{sgn}(S)}{\lambda_{1} g_{1}+\beta_{1} g_{2}},
\end{array}\right.
$$

which leads to

$$
\frac{d V}{d t}=S \cdot \dot{S}=-\left(k S^{2}+\eta S \operatorname{sgn}(S)\right) \leq 0 .
$$

Thus, the control signal for the SIMO under-actuated system is aggregated by

$$
\begin{aligned}
u & =u_{e q 1}+u_{s \mathrm{w} 1}+u_{e q 2}+u_{s \mathrm{w} 2} \\
& =\frac{\lambda_{1}\left(f_{1}(X)+c_{1} x_{2}\right)+\beta_{1}\left(f_{2}(X)+c_{2} x_{4}\right)+k S+\eta \operatorname{sgn}(S)}{-\lambda_{1} g_{1}(X)-\beta_{1} g_{2}(X)} .
\end{aligned}
$$

In order to minimize the chattering issue, a saturation function is proposed to replace the sign function in (26), where

$$
\operatorname{sat}(S)= \begin{cases}\operatorname{sgn}(S) & \text { if }|S|>1 \\ S & \text { if }|S| \leq 1\end{cases}
$$

That is, the control signal in (26) can be rewritten by

$$
\begin{aligned}
u= & -\frac{\lambda_{1} f_{1}(X)+\beta_{1} f_{2}(X)+\lambda_{1} c_{1} e_{2}+\beta_{1} c_{2} e_{4}}{\lambda_{1} g_{1}(X)+\beta_{1} g_{2}(X)} . \\
& -\frac{\lambda_{1} \ddot{x}_{d}+k S+\eta \operatorname{sat}(S)}{\lambda_{1} g_{1}(X)+\beta_{1} g_{2}(X)} .
\end{aligned}
$$

\section{B. Adaptive Law}

It can be seen in the analysis in Section III-A that if both the parameters $\lambda_{1}$ and $\beta_{1}$ in (22) are kept constant, it causes vibration in the control system. In order to address this issue, in this work we propose to adaptively adjust these parameters over time by the use of a fuzzy system. For the simplicity purpose, we define

$$
\beta_{1}=k \lambda_{1}
$$

which allows the fuzzy system to learn one parameter rather than two at one particular time. It is noticed that the factor
TABLE I: FUZZY INFERENCE FOR AHSMC

\begin{tabular}{|c|c|c|c|c|}
\hline \multicolumn{2}{|c|}{$\lambda_{1}$} & \multicolumn{3}{|c|}{$e_{1}$} \\
\cline { 3 - 5 } & & -1 & 0 & 1 \\
\hline \multirow{2}{*}{$\dot{e}_{1}$} & 1 & 0 & -1 & -2 \\
\cline { 2 - 5 } & 0 & 1 & 0 & -1 \\
\cline { 2 - 5 } & -1 & 2 & 1 & 0 \\
\hline
\end{tabular}

$k$ can be predefined. More interestingly, varying $k$ leads to change of the sliding surfaces. And if $k$ is properly chosen, the control system states move on the sliding surfaces to converge to zero, which effectively minimizes the chattering phenomena in the SIMO under-actuated systems.

We now investigate how to construct the membership functions and rule base for the two typical systems including the inverted pendulum cart and overhead crane.

The membership functions for the input parameters of the 2D inverted pendulum cart and 2D overhead crane systems, where each input parameter has a three triangle fuzzy set, are presented by

$$
\left[\begin{array}{lllll}
-2 & -1 & 0 & 1 & 2
\end{array}\right]
$$

which corresponds to

$$
\left[\begin{array}{lllll}
c_{1} & c_{2} & c_{3} & c_{4} & c_{5}
\end{array}\right]=\left[\begin{array}{lllll}
3 & 2.5 & 2 & 2.5 & 3
\end{array}\right]
$$

for the $2 \mathrm{D}$ inverted pendulum cart system and

$$
\left[\begin{array}{lllll}
c_{1} & c_{2} & c_{3} & c_{4} & c_{5}
\end{array}\right]=\left[\begin{array}{lllll}
10 & 9.25 & 7.5 & 9.25 & 10
\end{array}\right]
$$

for the 2D overhead crane. More importantly, the rule base is also constructed and summarized in Table I.

\section{Demonstrated RESUlts AND Discussions}

In order to demonstrate effectiveness of our proposed approach, adaptive fuzzy hierarchical sliding mode controller (AFHSMC), in controlling a class of SIMO under-actuated robotic systems, we conducted the experiments in the synthetic simulation environment for the two typical SIMO underactuated robotic systems including the $2 \mathrm{D}$ inverted pendulum cart and 2D overhead crane. The obtained results are presented in this section. It is noticed that the control performance is measured how effectively the cart in the pendulum system and the trolley in the overhead crane can reach the desired positions while the swing angles, which are between the inverted pendulum and hoisting cable and the vertical line, are minimized.

\section{A. 2D Inverted Pendulum Cart System}

In the experiments of the 2D inverted pendulum cart, it was assumed that some parameters of the system as presented in Section II-A were known. Those parameters are summarized in Table II.

Moreover, in order to verify robustness of the closed-loop control system given external disturbances, in the simulations we injected a noise with amplitude of $2(\mathrm{~N})$ into the system after it started $3(\mathrm{~s})$. The simulation results in the experiments with and without considering the external disturbance are 
TABLE II: PARAMETERS OF SIMO SYSTEMS.

\begin{tabular}{cc}
\hline Inverted pendulum & Overhead crane \\
\hline$m_{0}=1(\mathrm{~kg})$ & $M_{0}=6(\mathrm{~kg})$ \\
$m=0.1(\mathrm{~kg})$ & $m_{c}=3(\mathrm{~kg})$ \\
$l_{c}=1(\mathrm{~m})$ & $l=1(\mathrm{~m})$ \\
$c_{1}=1$ & $c_{1}=0.01$ \\
$c_{2}=5$ & $c_{2}=3$ \\
$k=5$ & $k=3.5$ \\
$\eta=10$ & $\eta=3.85$ \\
$g=9.81\left(\mathrm{~m} / \mathrm{s}^{2}\right)$ & $g=9.81\left(\mathrm{~m} / \mathrm{s}^{2}\right)$ \\
\hline
\end{tabular}

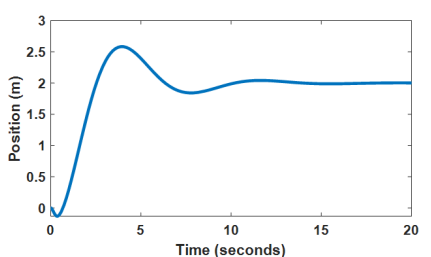

(a)

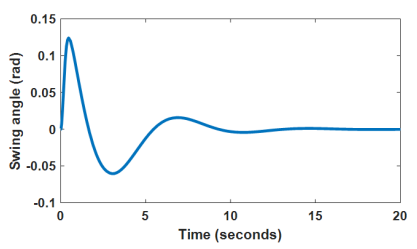

(c)

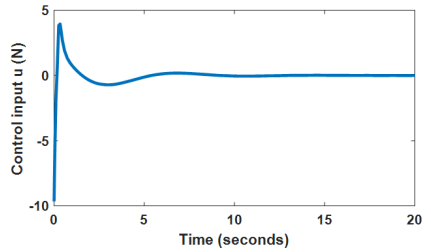

(e)

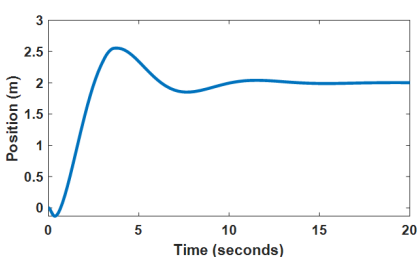

(b)

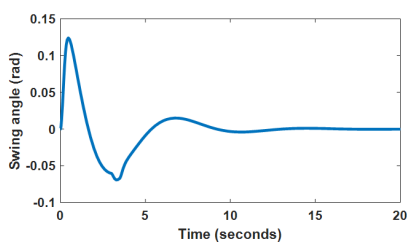

(d)

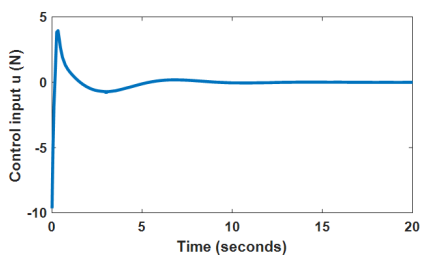

(f)
Fig. 1: The control performance on the 2D inverted pendulum cart system considered without (left column) and with (right column) the external disturbances.

illustrated in Fig. 1, where Figures 1a, 1c and 1e are the obtained results without considering the external noise while Figures $1 \mathrm{~b}, 1 \mathrm{~d}$ and $1 \mathrm{f}$ are the obtained results when the system was under influence of the external disturbance. It can clearly seen that in the both scenarios, the cart effectively reached to the desired position of $2(\mathrm{~m})$ and the swing angle considerably converged to the ideal condition of 0 (rad) after 10 (s). That is, stability of the inverted pendulum cart system is guaranteed regardless the external condition in the environment.

More importantly, we also implemented the standard HSMC algorithm in the 2D inverted pendulum cart system with the purpose of comparing with our proposed approach. The results obtained by our proposed algorithm and the standard HSMC law are demonstrated in Fig. 2, where we summarize not only the position and velocity of the cart but also the swing angle and angular velocity of the inverted pendulum. It is noted that in the standard HSMC algorithm it was assumed that the system parameters were deterministic (though it is not

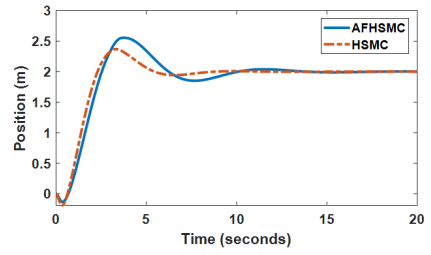

(a) Cart position

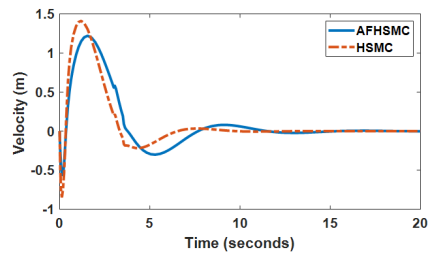

(c) Cart velocity

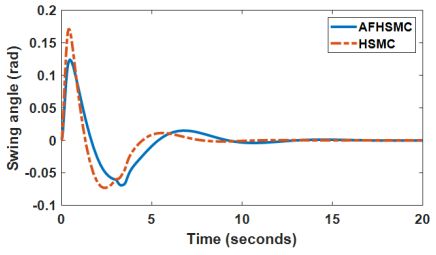

(b) Inverted pendulum swing angle

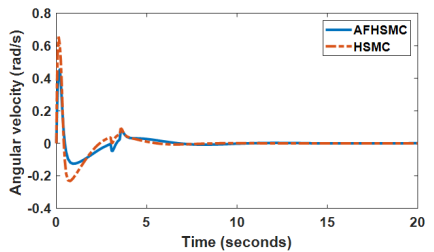

(d) Inverted pendulum swing angular velocity

Fig. 2: The control performance on the 2D inverted pendulum cart system obtained by our proposed approach as compared with that obtained by the standard HSMC algorithm.

realistic) while in our algorithm the system parameters were adaptively inferred by the fuzzy logic. However, the results obtained by our method are highly comparable with those obtained by the ideal standard HSMC scheme.

\section{B. 2D Overhead Crane}

To consolidate efficacy of our proposed algorithm in controlling a class of SIMO under-actuated robotic systems, we conducted further experiments on another typical 2D overhead crane. The parameters employed to simulate the 2D overhead crane and the controller are summarized in Table II.

Akin to the 2D inverted pendulum cart system, we first conducted the experiments considering that the environment had no noise. After that, in order to verify stability of the control performance in the 2D overhead crane, we intentionally influenced the gantry crane by an external disturbance of $15(\mathrm{~N})$ after the system started 5 (s) for a period of 2 (s). As expected, the proposed controller responded quite well to the external noise and kept the system very robust. The obtained results shown in Fig. 3 for the both cases without (left column) and with (right column) the external disturbance prove stability of the system regardless noises.

Comparison between our proposed algorithm and the standard HSMC approach were also conducted and the results are illustrated in Fig. 4. Not surprisingly the standard HSMC law outperformed our proposed technique a little bit since it was supposed the parameters in the HSMC scheme were known though determining the parameters in the nonlinear and highly uncertain SIMO under-actuated robotic systems is impractical. Hence, the results obtained by the standard HSMC law can be considered as the ideal. Nonetheless, the results obtained by our approach where its parameters are approximately and adaptively inferred by the fuzzy logic are highly comparable with the ideal. 


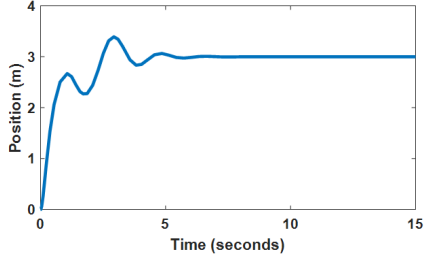

(a)

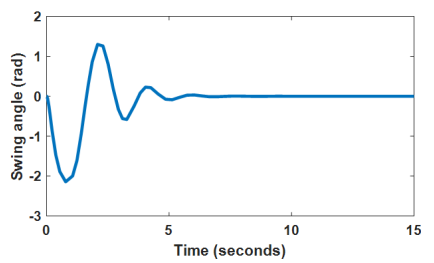

(c)

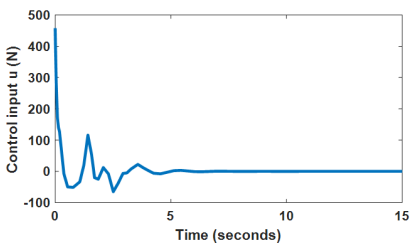

(e)

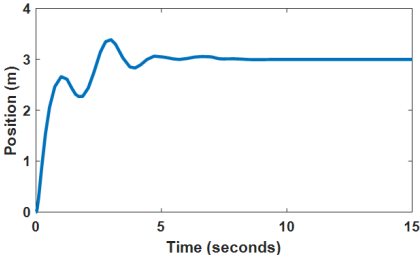

(b)

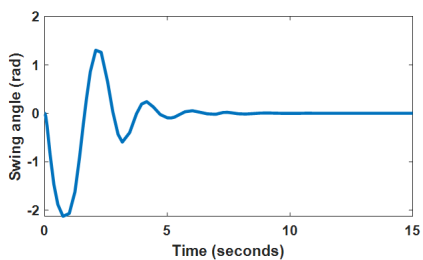

(d)

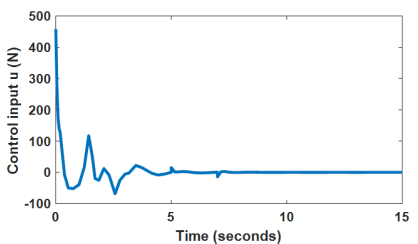

(f)
Fig. 3: The control performance on the 2D overhead crane considered without (left column) and with (right column) the external disturbances.

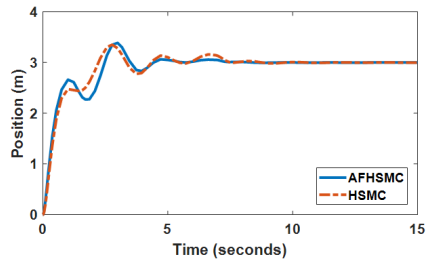

(a) Trolley position

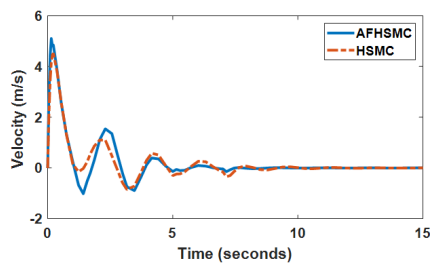

(c) Trolley velocity

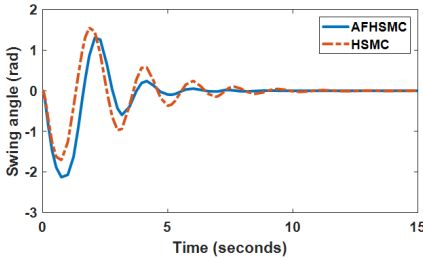

(b) Swing angle

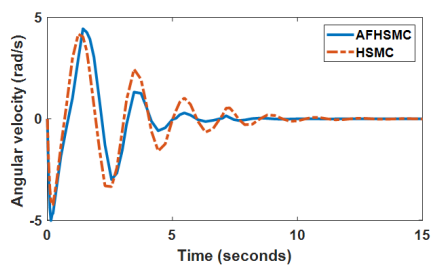

(d) Swing angular velocity
Fig. 4: The control performance on the 2D overhead crane obtained by our proposed approach as compared with that obtained by the standard HSMC algorithm.

\section{CONCLUSIONS}

The paper has introduced an efficient approach for controlling a class of SIMO under-actuated robotic systems, where a controller is established by the use of the HSMC law. Moreover, it has been proposed to exploit the fuzzy logic mechanism to infer the uncertain, nonlinear and unknown parameters of the system. The proposed algorithm was then validated in the two typical SIMO under-actuated systems including the $2 \mathrm{D}$ inverted pendulum cart and $2 \mathrm{D}$ overhead crane. The obtained results have demonstrated effectiveness of the proposed method.

\section{REFERENCES}

[1] M. W. Spong, "Underactuated mechanical systems," in Control Problems in Robotics and Automation, B. Siciliano and K. P. Valavanis, Eds. Berlin, Heidelberg: Springer, 1998, pp. 135-150.

[2] B. He, S. Wang, and Y. Liu, "Underactuated robotics: A review," International Journal of Advanced Robotic Systems, vol. 16, no. 4, p. $1729881419862164,2019$.

[3] M. J. Mahmoodabadi and H. K. Haghbayan, "An optimal adaptive hybrid controller for a fourth-order under-actuated nonlinear inverted pendulum system," Transactions of the Institute of Measurement and Control, vol. 42, no. 2, pp. 285-294, 2020.

[4] H. X. Le, L. Nguyen, and K. Thiyagarajan, "A dynamic surface controller based on adaptive neural network for dual arm robots," in 2020 15th IEEE Conference on Industrial Electronics and Applications (ICIEA), 2020, pp. 555-560.

[5] T. V. Nguyen, N. H. Thai, H. T. Pham, T. A. Phan, L. Nguyen, H. X. Le, and H. D. Nguyen, "Adaptive neural network-based backstepping sliding mode control approach for dual-arm robots," Journal of Control, Automation and Electrical Systems, vol. 30, no. 4, pp. 512-521, Aug 2019.

[6] D. T. Pham, T. V. Nguyen, H. X. Le, L. Nguyen, N. H. Thai, T. A. Phan, H. T. Pham, A. H. Duong, and L. T. Bui, "Adaptive neural network based dynamic surface control for uncertain dual arm robots," International Journal of Dynamics and Control, vol. 8, no. 3, pp. 824-834, Sep 2020.

[7] U. T. T. Hoang, H. X. Le, N. H. Thai, H. V. Pham, and L. Nguyen, "Consistency of control performance in $3 \mathrm{~d}$ overhead cranes under payload mass uncertainty," Electronics, vol. 9, no. 4, p. 657, Apr 2020. [Online]. Available: http://dx.doi.org/10.3390/electronics9040657

[8] V.-A. Le, H.-X. Le, L. Nguyen, and M.-X. Phan, "An efficient adaptive hierarchical sliding mode control strategy using neural networks for $3 \mathrm{~d}$ overhead cranes," International Journal of Automation and Computing, vol. 16, no. 5, pp. 614-627, Oct 2019.

[9] H. X. Le, A. V. Le, and L. Nguyen, "Adaptive fuzzy observer based hierarchical sliding mode control for uncertain 2d overhead cranes," Cyber-Physical Systems, vol. 5, no. 3, pp. 191-208, 2019.

[10] X. Weimin, Z. Xiang, L. Yuqiang, Z. Mengjie, and L. Yuyang, "Adaptive dynamic sliding mode control for overhead cranes," in 2015 34th Chinese Control Conference (CCC), 2015, pp. 3287-3292.

[11] W. Wang, J. Yi, D. Zhao, and D. Liu, "Design of a stable slidingmode controller for a class of second-order underactuated systems," IEE Proceedings - Control Theory and Applications, vol. 151, no. 6, pp. 683690, 2004.

[12] L. A. Zadeh, "Is there a need for fuzzy logic?" Information Sciences, vol. 178, no. 13, pp. 2751 - 2779, 2008.

[13] M. Yue, C. An, Y. Du, and J. Sun, "Indirect adaptive fuzzy control for a nonholonomic/underactuated wheeled inverted pendulum vehicle based on a data-driven trajectory planner," Fuzzy Sets and Systems, vol. 290, pp. 158 - 177, 2016, theme: Control Engineering and Applications.

[14] A. I. Roose, S. Yahya, and H. Al-Rizzo, "Fuzzy-logic control of an inverted pendulum on a cart," Computers \& Electrical Engineering, vol. 61, pp. $31-47,2017$. 